\title{
New aspects of line-profile variability in P Cygni's optical spectrum
}

\author{
N. Markova \\ National Astronomical Observatory, P.O. Box 136, 4700 Smoljan, Bulgaria
}

Received March 23, 1999; accepted March 14, 2000

\begin{abstract}
High-resolution photographic spectra as well as high $\mathrm{S} / \mathrm{N}$ ratio $\mathrm{CCD}$ spectral observations of $\mathrm{P}$ Cygni are analyzed in terms of line-profile variability (lpv). Four different kinds of lpv are established: systematic variability in the absorption troughs of low and intermediate excitation lines due to propagating Discrete Absorption Components (DACs); "swaying" variability consisting of continuous modulations in velocity and intensity of the absorption cores and emission peaks of lines of intermediate and high excitation; red-emission-wing variability due to travelling "bumps", and long-term (LT) variability in $\mathrm{HI}$ and HeI lines of relatively large optical depth.
\end{abstract}

DAC propagation is a slow variation of $\mathrm{P}$ Cygni's stellar wind. The components probably originate from large-scale, high-density (low-excitation) perturbation(s) which develop in a relatively outer part of the wind $\left(V \geq 0.41 V_{\text {inf }}\right)$ but appear to be maintained, in some indirect way, by photospheric processes. The geometry of the structures is not yet clear but they could be either spherically symmetric or curved, like kinks.

The "swaying" variability manifests itself by modulations in position and intensity of the absorption cores and emission peaks of almost all lines in the optical. Simultaneous variations in emission and absorption linestrength were also observed. The modulations are at least partially due to variations in the number density which affect all layers of the supersonic wind starting at its base up to layers where the Hff line forms $\left(0.18 \leq V \leq 0.95 V_{\text {inf }}\right)$. The phenomenon appears to be stable over many years, though on a variable time-scale. Suggestive evidence for a close relationship between the modulations and changes in the stellar brightness and temperature was found, indicating that the "swaying" variability is more likely coupled to processes in the photosphere. Non-radial pulsations (NRPs) either of g-mode or of s-mode oscillations are a possible cause for this variability.

The LT variability makes up a very slow pattern of variation in both the velocity of the absorption cores and the intensity of the emission peaks of the stronger $\mathrm{HI}$ and HeI lines. This variability is found only in the outer part of the wind ( $\left.V \leq 0.82 V_{\mathrm{inf}}\right)$. The nature of the LT variation is not known at present.

The red-emission-wing variability is localized in the high-velocity part of the emission lobes of P Cygni-type profiles, $+90 \leq V \leq+230 / 250 \mathrm{~km} \mathrm{~s}^{-1}$. This variability is presumably caused by outward propagating "bumps", but its exact nature is still unknown.

No indication for any clear relation between different kinds of lpv was found. Even when the variations operate in one and the same region of the wind (in velocity space) it is not obvious whether and how they interact. Stellar rotation does not seem to play a fundamental role in setting the time-scale of either the DAC-induced variability or the LT variability. The relationship between the "swaying" variability and the rotation is still not clear but it is possible that this variability is rotationally modulated.

The wind variability of $\mathrm{P}$ Cygni appears to be qualitatively similar to the wind variability of the early B-type supergiant HD64760 but different from the variability of O-type star winds.

Key words: stars: mass loss — stars: oscilations — line: profiles — stars: individual: P Cygni

\section{Introduction}

$\mathrm{P}$ Cygni is an early B-type supergiant classified variously as B1eq, B0-B1, B1p (de Jager 1980) or B1Ia+ (Lamers et al. 1983). Because of its spectral characteristics the star is considered the prototype of the P Cygni-type stars (PCT) as defined by Lamers (1986) and, also, along with $\eta$ Carina and $\mathrm{S}$ Dor, as one of the prototypes of the Luminous Blue Variables (LBVs). LBVs form a rather heterogeneous class of very massive, unstable stars located close to the Humphreys-Davidson limit on the HR diagram. The stars are characterized by high mass-loss rates, episodes of shell ejection and large eruptions. Though considered to be in a quiescent phase, the LBV P Cygni 
has demonstrated several aspects of wind activity such as line-profile variability (lpv) at UV and optical wavelengths; radio-flux variability (van den Oord et al. 1985; Skinner et al. 1997) and polarization variability (Hayes 1985; Taylor et al. 1991). The most widely studied is the lpv of optical lines, which manifests itself by variations in emission and absorption-line strength, by changes in velocity of absorption cores and emission peaks, as well as by the appearance of Discrete Absorption Components (DACs).

DACs are the most prominent feature of P Cygni's wind variability. For a long time these features were thought to be a permanent characteristic of the spectrum since they were readily recognized in almost all snapshot observations. In addition, DACs were detected not only in several unsaturated resonance and subordinate lines of several highly ionized species in the UV as well as in Hff which is the situation for other luminous OB stars (Prinja et al. 1992; Henrichs et al. 1994; Kaper et al. 1997) but also in a variety of lines of different excitation and ionization in the UV and optical domain (Israelian \& de Groot 1999). In view of this apparent ubiquity of the phenomenon the result reported by Stahl et al. (1994) was really a surprise: no DAC was found in any of the CCD spectra taken over the years 1990 to 1992 . Motivated by this finding Israelian et al. (1996) studied the variability of UV metal lines and found that these lines still showed DACs in 1990. To bring all these results into line one must assume, either that the majority of DACs detected in the optical by previous investigators were not real but due to unrecognised photographic noise, or that the activity of the mechanism that causes the appearance of DACs in the optical has changed and that, as a result, the components disappeared. It is also possible that for some reasons, e.g. relatively low spectral resolution $(R=12000)$, Stahl et al. failed to detect any DACs.

Another kind of lpv in $\mathrm{P}$ Cygni consists of variations in velocity of the absorption troughs of optical lines. Markova (1993) found from 1981 observations that high-excitation lines, such as OII, NII, SiIII, exhibited smooth variation in the position of the maximum absorption depth with an amplitude of 30 to $50 \mathrm{~km} \mathrm{~s}^{-1}$ and a peak-to-peak timescale of 100 to 120 days. Similar variations, but on the shorter time-scale of 50 to 60 days, were established by Kolka (1989, 1991) for weaker lines of HI and HeI in 1982 and 1989. Long-term observations suggest that variations on different time-scales may coexist in the lines (Stahl et al. 1994). The nature and the origin of this kind of lpv is not known at present.

Variations of up to 30 per cent in emission and absorption-line strength (i.e. equivalent width, EW) were measured on various occasions (Luud 1967a,b; de Groot 1969). Long-term observations with high time resolution (Markova 1986a, 1993; Stahl et al. 1994) showed that line-strength variations, at least in the strongest Balmer and HeI lines, do not seem to be accidental but follow a definite pattern of behaviour. Neither the origin of these variations nor how they affect other spectral lines formed deeper in P Cygni's wind, are known at present.

Thus, we see that our knowledge of lpv in P Cygni is far from complete. Most of the above-quoted studies share one and the same failure: they focus only on a few particular aspects of lpv in particular ions, e.g. DAC propagation, radial-velocity or line-strength variations, etc. For this reason, none of the investigators were able to make a direct comparison between different kinds of lpv in order to establish possible relationships between them and, thus, to look for the origin of the variability.

The purpose of our work is to investigate the temporal behaviour of a large sample of optical lines of various excitation and ionization in order to classify the different kinds of lpv, to specify their properties, and to search for possible relationships between them. Studying lines formed in different layers of $\mathrm{P}$ Cygni's wind simultaneously we hope to gain a better understanding of the nature and the origin of P Cygni's wind variability. Additionally, we aim to clarify the situation for the optical DACs in 1990. In Sect. 3.1 some characteristics of the DAC phenomenon specific to $\mathrm{P}$ Cygni are highlighted and the appearance of DACs in the 1990 photographic spectra is examined. In Sects. 3.2, 3.3 and 3.4 we elaborate on the simultaneous behaviour of different lines in the spectrum. In Sect. 4 we discuss the nature of the different kinds of lpv found, with special emphasis on the "swaying" variability and the DAC-induced variability. The relationship between the "swaying" variability and the photometric variability is examined. Several qualitative similarities between the wind variability of P Cygni and the B-type supergiant HD 64760 are noted.

\section{Observations and data reduction}

Our data sample consists of 45 CCD spectra published by Stahl et al. (1993) together with a further 10 photographic spectra obtained with the coud'e spectrograph of the $2 \mathrm{~m}$ telescope at the National Astronomical Observatory (Bulgaria). The spectra cover the period from May to December 1990. The spectral resolution is about 27000 in the blue and 18000 in the red photographic region. The resolution of the CCD observations is a factor of two lower. Additional information about the observational material and its reduction can be found in Stahl et al. (1991) and Markova (1986b).

\section{Line-profile variability at optical wavelengths}

To obtain a detailed picture of lpv in P Cygni's optical spectrum we studied the simultaneous behaviour of about 60 lines (permitted and forbidden) of different ions (such as NaI, CaII, HI, MgII, HeI, FeIII, NII, SiIII, SiIV, OII and SIII) over a period of 8 months in 1990 . 

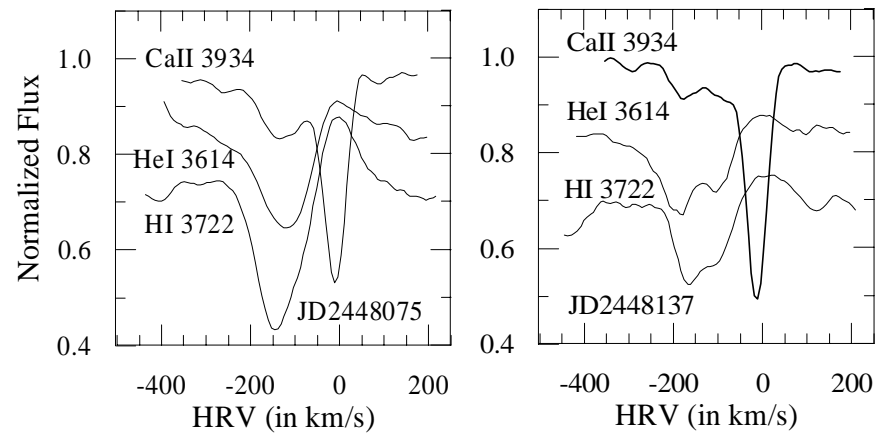

Fig. 1. Examples of clear line-splitting observed in the 1990 spectrum of P Cygni. The profiles have been offset vertically by an amount that provides better visibility. The horizontal axes are scaled in heliocentric radial velocity (HRV)

\subsection{DAC-induced variability}

Photographic spectral observations carried out by different investigators at different epochs suggest that Balmer and HeI lines appear to be the best indicator of DACs in P Cygni's optical spectrum (see Table 1 in Israelian \& de Groot 1999). This fact does not necessarily mean, however, that ALL lines of the two ions shows DACs at ALL observational epoch. Based on our own experience we can state that line-splitting can be confidently and frequently detected in Balmer lines with principal quantum number $9 \leq n \leq 15$ and in HeI lines from the $2^{1} \mathrm{~S}$ level $(\lambda \leq 3964 \AA)$. As an additional indicator of DACs, one may also use the K-line of CaII, MgII $\lambda 4481.14$ and HeI lines of:

- the triplet series $2^{3} \mathrm{P}-n^{3} \mathrm{D}$ with $\lambda \leq 3819 \AA$;

- the triplet series $2^{3} \mathrm{P}-n^{3} \mathrm{~S}$ with $\lambda \leq 4713 \AA$;

- the singlet series $2^{1} \mathrm{P}-n^{1} \mathrm{D}$ with $\lambda \leq 4387 \AA$.

With the above outlined in mind we examined the photographic spectra of our sample for the appearance of DACs and found that some of the listed lines indeed exhibited, at least occasionally, line-splitting. Line-splitting was also suspected in FeIII lines. Examples of lines with a distinct double structure in absorption are shown in Fig. 1. To find additional evidence for the existence of DACs in the 1990 spectra of P Cygni we studied the so-called "quotient" spectra derived by dividing individual photographic spectra by the spectrum obtained on the first night of the time series (JD 2448017), which was assumed - because of the lack of line-splitting and the relatively small width and strength of the absorption troughs - to represent a minimum-absorption template. This wavelengthby-wavelength renormalization effectively removes the underlying profile, thus emphasising small changes in line depth: parts of a profile that have excess absorption (or weakened emission) with respect to the corresponding "undisturbed profile" appear in the quotient spectrum as localized dips, while parts characterized by weakened absorption (or enhanced
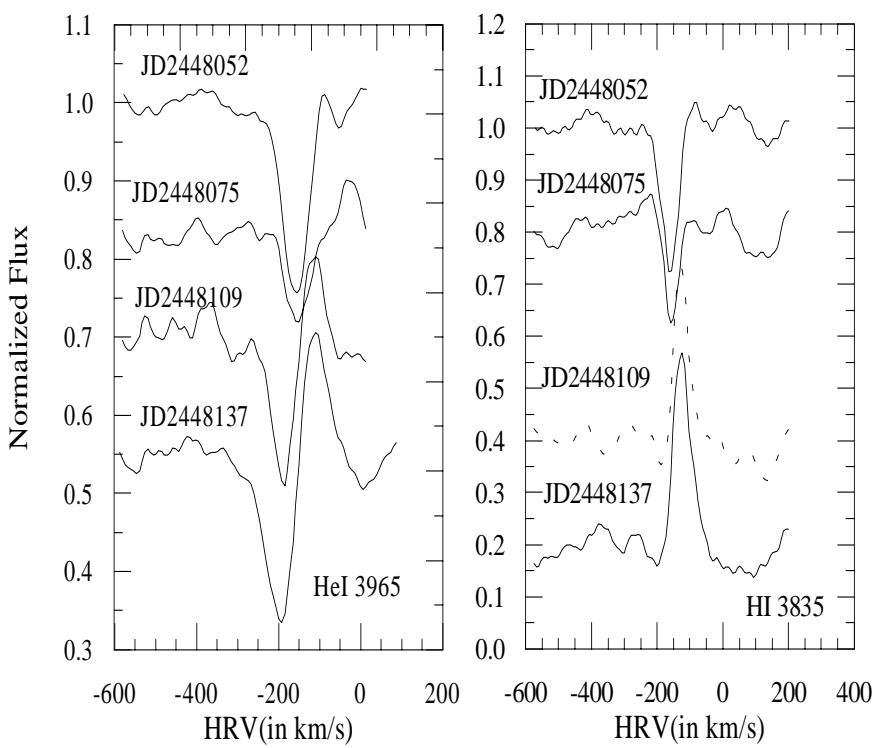

Fig. 2. Quotient spectra in the domain of $\mathrm{HI} \lambda 3835$ and HeI $\lambda 3965$. For better visibility successive spectra have been offset vertically and the HI $\lambda 3835$ spectrum on JD 2448109 is represented with a dashed line. Horizontal axes are scaled in heliocentric radial velocity (HRV)

emission) relative to the "undisturbed profile" appear as localized peaks. The quotient spectra of the lines showing DACs revealed the existence of excess absorption, whose position in the troughs varied with time. The spectra also show that the emission lobes of the lines did not exhibit variations connected with those in absorption. The quotient spectra in the domain of HI $\lambda 3835.38$ and HeI $\lambda 3964.73$ are displayed in Fig. 2. The appearance of a sharp "emission" bump, redward of the absorption enhancements is largely an artifact of the renormalization procedure.

The quotient spectra of the lines showing DACs were measured for the position of the dips (i.e. heliocentric radial velocity, HRV), for the full width at half the maximum intensity (FWHM) and for the strength (i.e. equivalent width, EW). The velocity of each feature was measured by bisecting the lower half part of its profile. The precision of the measurements is better than $3 \mathrm{~km} \mathrm{~s}^{-1}$. The other two parameters, i.e. FWHM and EW, were estimated by means of least-squares Gaussian fits to the observed profiles. The accuracy of the individual determinations is limited by uncertainties in both the fitting procedure and the definition of the level of the continuum (typically of the order of 1 to 2 percent of the continuum flux). The estimated error is about a few hundredths of an $\AA$ in EW and 10 to $15 \mathrm{~km} \mathrm{~s}^{-1}$ in FWHM. The measurements showed that:

(i) within each spectrum there exists an essentially one-to-one correspondence (in velocity and width) between the enhancements: the rms deviations in the 
velocity and the FWHM of the components amounted to less than 5 and $8 \mathrm{~km} \mathrm{~s}^{-1}$, respectively;

(ii) during the studied period the velocity of the enhancements increased from about 150 to about $190 \mathrm{~km} \mathrm{~s}^{-1}$;

(iii) the width of the enhancements seems to remain constant (within the error) around a mean value of $69 \pm$ $10 \mathrm{~km} \mathrm{~s}^{-1}$;

(iv) the strength (i.e. the EW) of the enhancements varied by up to $50 \%$ with no hint of any systematics.

The "classical" picture we have of DAC behavior is that broad absorption components appear at low velocity (one and the same for lines of different ions) and evolve towards higher velocity while narrowing. Thus we see that the behavior of the observed absorption enhancements only partially resembles the behavior of "classical" DACs. The fact that both the width and the strength of the enhancements do not show variations typical for bluewardmigrating DACs, i.e. do not decrease with increasing velocity, indicates, either that the components do not belong to one and the same DAC episode, or that another variable phenomenon, different from the DAC-induced variability, exists and modifies the characteristics of the line profiles.

Unfortunately, our data sample has large gaps between data points and is not extensive enough to allow any specification in terms of different DAC episodes to be made. By chance, our photographic observations partly overlap the UV observations studied by Israelian et al. (1996). Combining the velocity measurements obtained for DACs in the optical (present study) with measurements derived for DACs in the UV we found that the two datasets agreed quite well in a sense that they defined a smooth curve of gradually increasing velocity (Fig. 3). This result suggests that the studied features are likely to belong to one and the same DAC episode. The observed duration of the episode is about 200 days. A linear interpolation of the data presented in Fig. 3 gives a mean acceleration of 0.37 and $0.10 \mathrm{~cm} \mathrm{~s}^{-2}$ for DACs with velocities between 150 and $-190 \mathrm{~km} \mathrm{~s}^{-1}$ and between -190 and $-200 \mathrm{~km} \mathrm{~s}^{-1}$, respectively. Notice that the phase of slower acceleration appears to consist of two subphases: one of almost zero DAC acceleration (between JD 2448120 and JD 2448230) followed by another with futher DAC acceleration (after JD 2448230). The separation does not seem to be an artifact of bad data sampling since similar effect has been observed during previous DAC episodes (Markova 1986b, Fig. 1 components Nos. 2 and 6; Fig. 3). The possibility that DACs may not accelerate continuously during their life-time is really unexpected and need futher investigation. It may well be, however, that the observed effect has no physical cause but reflects some kind of observational difficulty, e.g. unresolved blends between consecutive DACs.

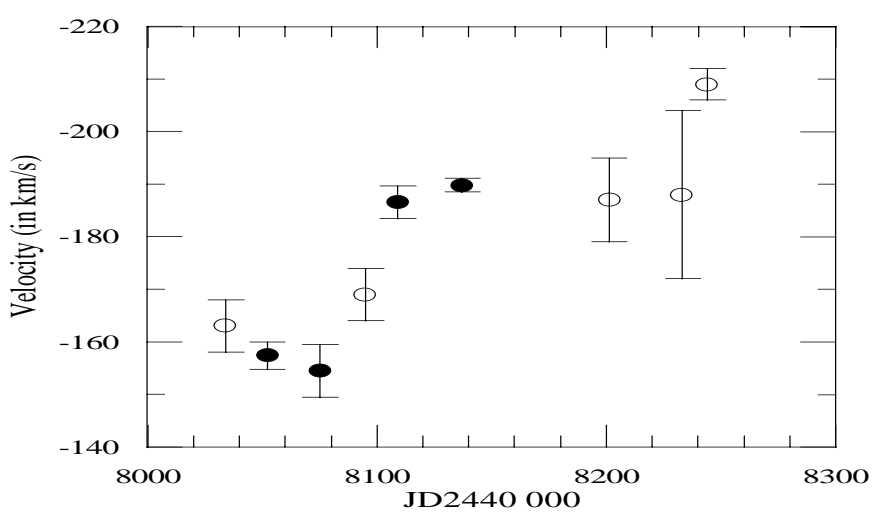

Fig. 3. Velocity curve for absorption enhancements in the wind of P Cygni in 1990. Velocities are measured with respect to the Sun. Filled circles denote measurements in the optical (i.e. our data) while open circles - measurements in the UV (Israelian et al. 1996). Error bars are also drawn

\section{2. "Swaying" variability}

Most lines in P Cygni's optical spectrum do not show clear line-splitting but exhibit significant variations in their profiles (Markova 1993; Stahl et al. 1994). In order to specify the properties of this variability we examined the temporal behavior of all lines in our sample simultaneously and looked for possible relationships between different kinds of lpv. (Note that because of the short-wavelength cutoff of the CCD observations the behaviour of some lines, e.g. the higher members of the Balmer and HeI series, remains beyond the scope of this analysis.) Figure 4 (left panels) shows representative profiles of lines of different ions. Profiles are normalized to the continuum. The wavelengths of each of the transitions and the Julian Date of observation are indicated. It is immediately apparent from this figure that different lines show qualitatively similar lpv, expressed mainly by changes in line absorption over a wide range of velocities. Variations in the width and the blue-edge velocity of the troughs are also apparent. In Fig. 4 (right panels) the distribution of the variability (by means of rms deviations, $\sigma_{\lambda}$ ) across the line profiles is shown as a function of velocity. The quantity $\sigma_{\lambda}$ was calculated using the expression

$\sigma_{\lambda}^{2}=\sum_{i=1}^{N} \frac{\left[f_{i}(\lambda)-F(\lambda)\right]^{2}}{(N-1) f(\lambda)}$

where $f_{i}(\lambda)$ is the normalized intensity in the $i^{\text {th }}$ of $N$ spectra, and $F(\lambda)$ is the mean spectrum. In our case $N=46$. This formulation, first used by Prinja et al. (1996), provides a good approximation to the Temporal Variance Spectrum (TVS) described by Fullerton et al. (1996), under the additional assumption that the noise is dominated by photon noise and is nearly the same for each spectrum in the time series. The results obtained show that:

(i) the variability is dominated by changes in the troughs, which extend from zero velocity (in lines of higher 

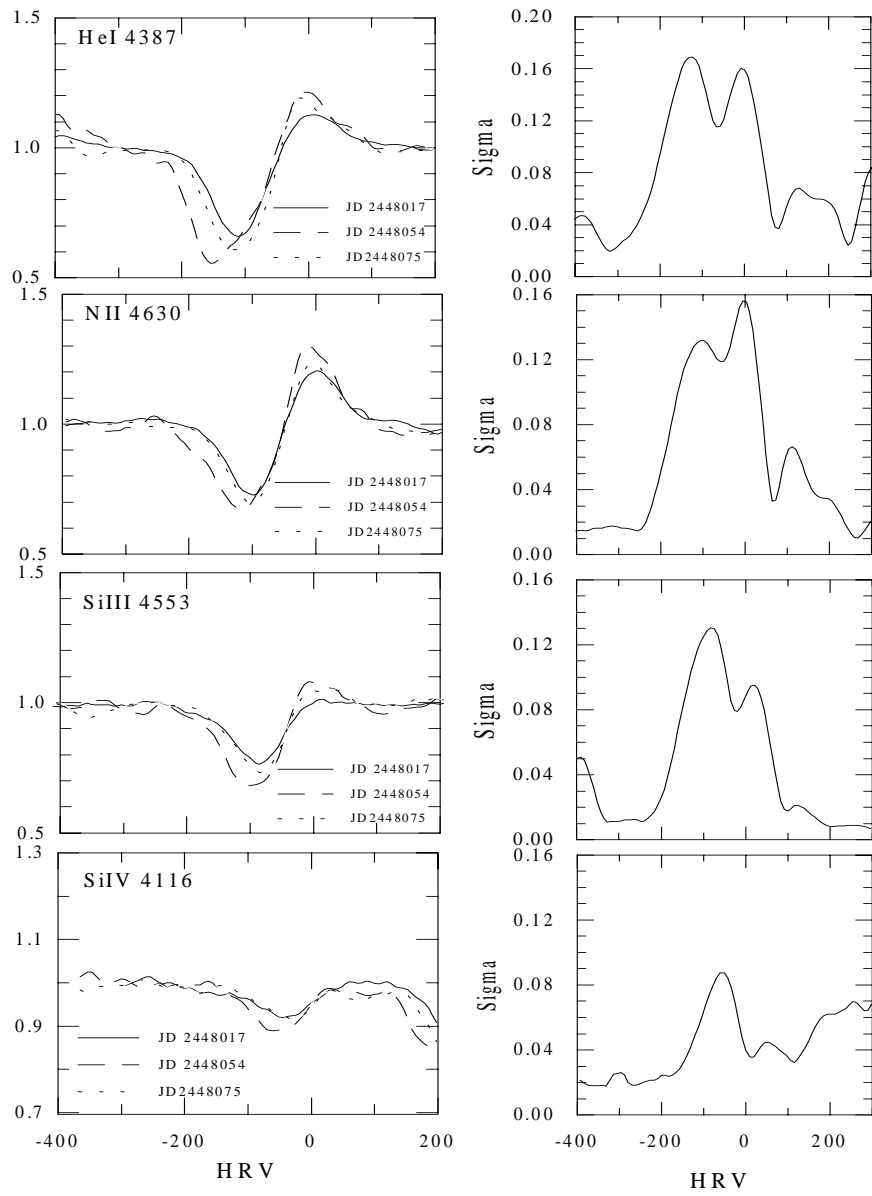

Fig. 4. Sets of observational profiles of optical lines of various ions from the 1990 data (left panels). The panels on the right show the rms deviations for the entire data set as a function of velocity in each of the lines shown on the left

excitation such as SiIV) to about -250 to $-300 \mathrm{~km} \mathrm{~s}^{-1}$ (in the strongest HI and HeI lines). Because we did not detect any DACs with velocity lower than $140 \mathrm{~km} \mathrm{~s}^{-1}$ (Sect. 3.1), we suggested that another form of lpv, in addition to the DAC-induced variability, exists and affects the deeper layers of P Cygni's wind;

(ii) for lines with $\mathrm{P}$ Cygni-type profiles the variations also extend throughout the emission region with two welldefined peaks of maximum activity situated, respectively, at about +100 to $+130 \mathrm{~km} \mathrm{~s}^{-1}$, and at about -20 to $+20 \mathrm{~km} \mathrm{~s}^{-1}$.

To quantify the observed variability we measured, using a fitting procedure with Gaussians, the absorption troughs and the emission lobes of the lines for the magnitude and position (i.e. heliocentric radial velocity, HRV) of the maximum absorption depth or emission excess and for equivalent width. The measurements are partly illustrated in Figs. 5 to 10. In particular, Fig. 5 emphasizes the similarity in the velocity variability of lines of different ions and optical depth. Note that, in contrast to the DAC-induced variability which is characterized by a grad-

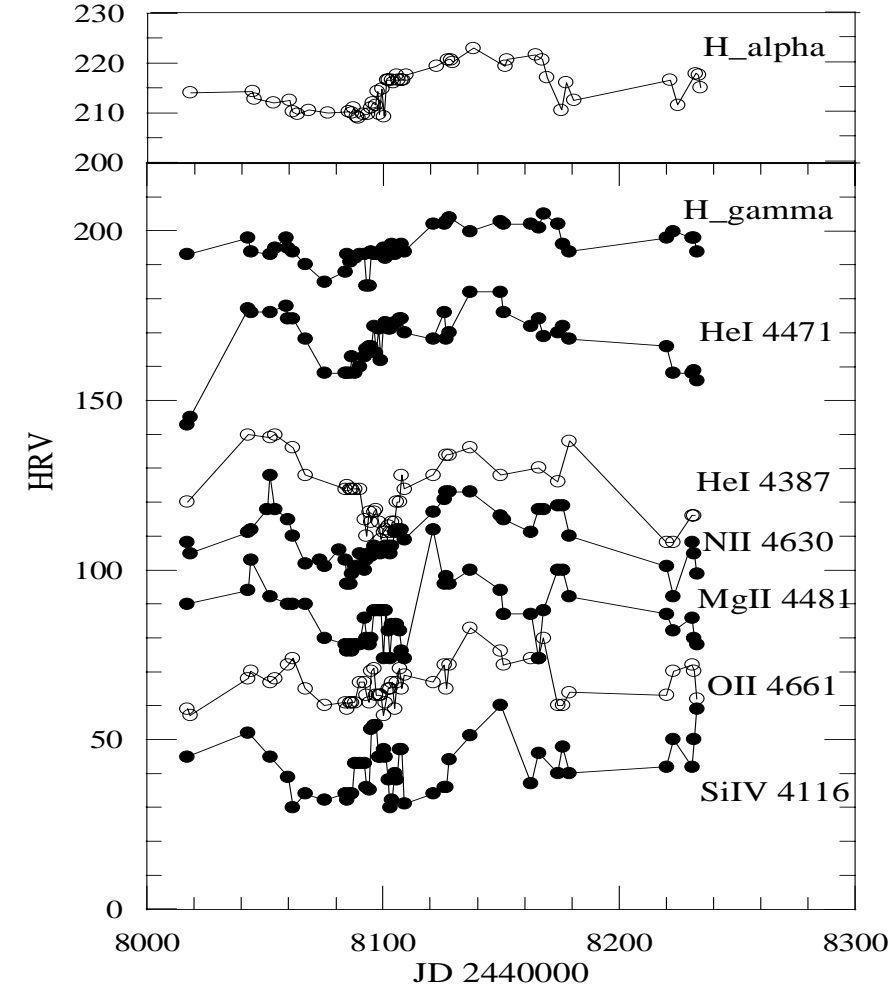

Fig. 5. Variations in the velocity of the absorption cores of lines of various ions. Vertical axes are scaled in heliocentric radial velocity (HRV). All velocities are negative

ual increase in velocity, this sort of variability consists of fluctuations above and below a mean velocity level specific for each line. The amplitude of the fluctuations ranges from about 10 to about $30 \mathrm{~km} \mathrm{~s}^{-1}$ with a tendency to increase with decreasing Total Excitation Energy (TEE = excitation potential + ionization potential). No clear evidence for a time-lag in the variation of lines of different ions was found. It is worth noting that the mean velocity around which the position of the absorption core of the SiIV $\lambda 4116$ line varies amounts to $-20 \pm 8 \mathrm{~km} \mathrm{~s}^{-1}$ in the star's rest frame, i.e. it is practically equal to the isothermal sound speed $\left(-19 \mathrm{~km} \mathrm{~s}^{-1}\right)$. This result indicates that the "swaying" variability affects the deepest layers of the supersonic wind down to its base. On the other hand, as far as the strongest lines of $\mathrm{HI}$ and $\mathrm{HeI}$ also exhibit variations in velocity similar to that in high-excitation lines (upper panel in Fig. 5), we suggest that upper wind layers - at least up to about $14 R_{*}$, where the $\mathrm{H} \alpha$ line forms (Vakili et al. 1997) - also respond to the perturbation(s) that causes the "swaying" variability of the inner wind.

Apart from modulations in the velocity of the absorption cores, we have also observed changes in the magnitude of the maximum absorption flux, $F_{\max }(\mathrm{abs})=1-\operatorname{Min}\left(F_{\lambda} / F_{\mathrm{c}}\right)$, and emission excess, $F_{\max }(\mathrm{em})=\operatorname{Max}\left(F_{\lambda} / F_{\mathrm{c}}\right)$, of the lines, as well as variations in emission and absorption strength, EW. Variations in the position of the emission peaks were also found. 

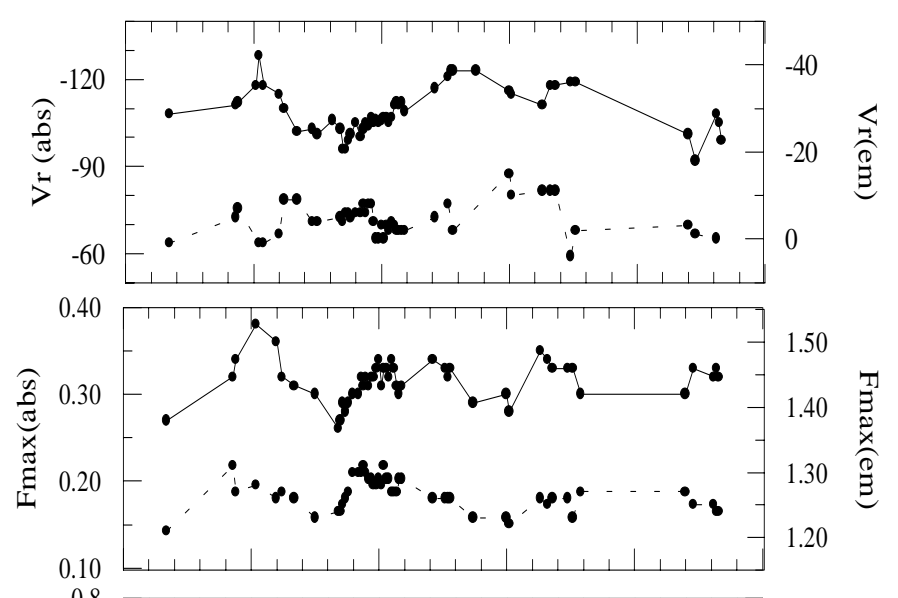

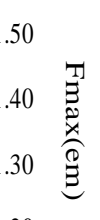

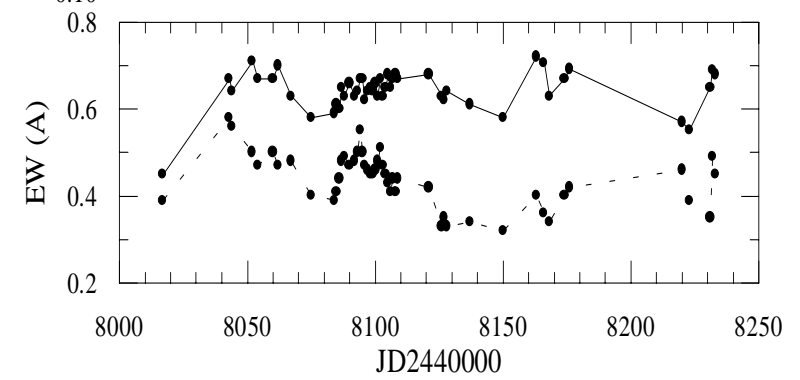

Fig. 6. Variability of measured properties of the absorption trough (circles joined by a solid line) and the emission lobe (circles joined by a dashed line) of the NII $\lambda 4630$ line. $F_{\max }(\mathrm{abs})$ $=1-\operatorname{Min}\left(F_{\lambda} / F_{\mathrm{c}}\right)$ and $F_{\max }(\mathrm{em})=\operatorname{Max}\left(F_{\lambda} / F_{\mathrm{c}}\right)$. For further explanation see the text

The measurements show that the variability in question consists of fluctuations around a mean level. Different lines seem to behave in a similar way. Modulations in absorption resp. emission EW are usually correlated with changes in $F_{\max }(\mathrm{abs})$ resp. $F_{\max }(\mathrm{em})$. The variability (in flux and strength) of the emission lobes is similar to but not identical to the variability of the troughs. Although the established line-flux (strength) modulations are usually small - for example, in the case of NII $\lambda 4630$ the rms deviations in emission and absorption EW amount to 0.06 and $0.05 \AA$ respectively, while the $\mathrm{rms}$ deviation in the $F_{\max }(\mathrm{abs})$ and $F_{\max }(\mathrm{em})$ is only $2 \%$ of $F_{\mathrm{c}}$ - they must be genuine since their amplitudes are larger than the accuracy of the individual determinations. In particular, the modulations cannot be artifacts of inconsistent continuum placement. Otherwise one would observe a distinct anticorrelation between variations in emission and in absorption. The pattern of variations in the NII $\lambda 4630$ line is illustrated in Fig. 6.

The present data are obviously insufficient to perform a detailed time-series analysis and to find out whether the established "swaying" variability is periodic or not. On the other hand, they clearly indicate that the phenomenon is recurrent. During the period studied we observed two cycles in the velocity modulations (the second one is incomplete) with a peak-to-peak time-scale of about 100 days,

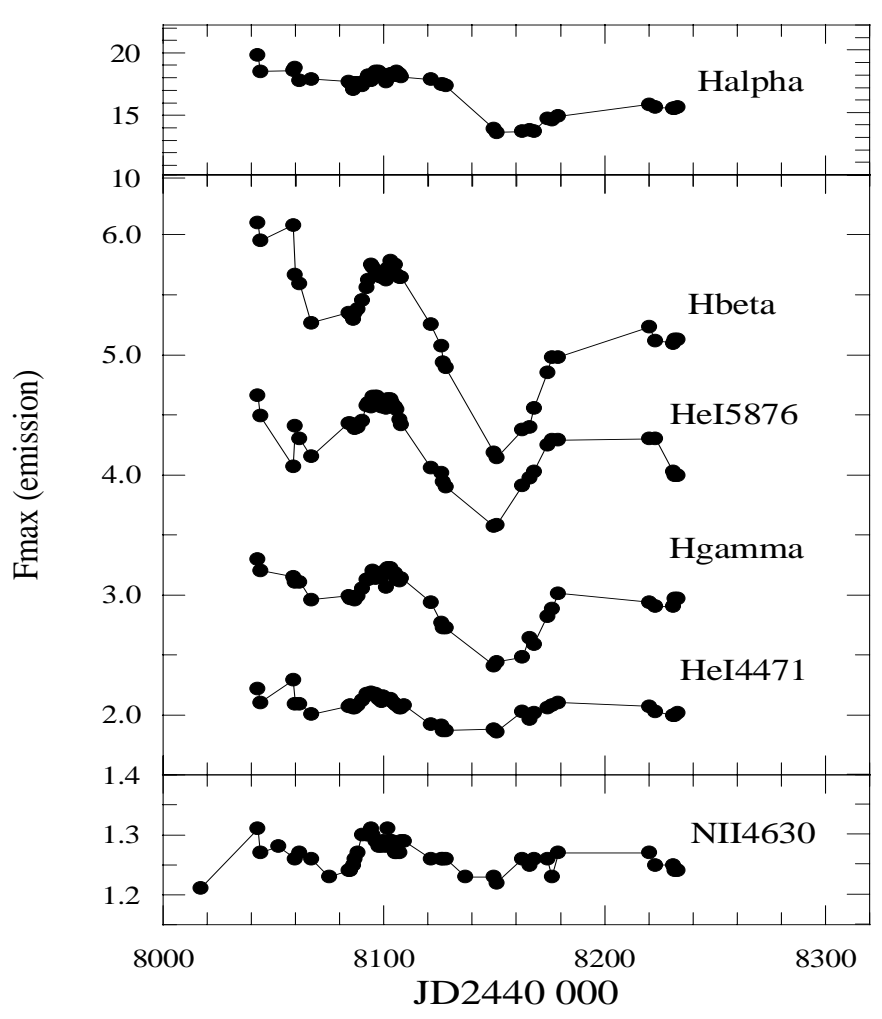

Fig. 7. Variations in the peak emission of lines formed at different optical depths. The pattern of variations in the strongest Balmer and HeI lines is obviously different from that in NII $\lambda 4630$. In the former lines line-flux modulations are superimposed on a LT variation in $F_{\max }(\mathrm{em})$, while in NII $\lambda 4630$ only modulations in $F_{\max }(\mathrm{em})$ have been observed. Note the apparent decrease in the amplitude of the LT variation with decreasing optical depth. For further explanation see the text

and almost three cycles in the line-flux (strength) modulations, with peak values separated by about 60 days. The estimates obtained, although rather uncertain, imply that the time-scale for modulations in velocity of the absorption cores might differ from the time-scale associated with modulations in emission and absorption-line strength (flux).

\subsection{Long-term variability and red-emission-wing variability due to traveling bumps}

Stahl et al. (1994) published radial-velocity and emissionpeak-intensity measurements of the strongest $\mathrm{HI}$ and HeI lines in $\mathrm{P}$ Cygni's optical spectrum. The presented data suggest the existence of a slow variation in both the velocity of the cores and the intensity of the peaks of the lines. Analyzing the data, we found in addition that:

(i) the LT variation in velocity was anti-correlated with the LT variation in emission-peak intensity in the sense that when the size of the emission peak increased the absorption core moved to longer wavelength; 
(ii) the amplitude of the LT variation, both in velocity and intensity, tended to decrease with decreasing line optical depth thus indicating that, whatever the cause of the LT variability, its activity presumably decreased inwards without reaching the deepest layers of the wind. This assumption was confirmed by our measurements, which showed that neither weaker lines of HI and HeI nor lines of higher-excited ions experienced variation in velocity or emission-peak intensity similar to the LT variability.

The effect of inward-decreasing amplitude of the LT emission-peak-intensity variation is illustrated in Fig. 7, where the limiting case of a line with and without LT variability is represented, respectively, by $\mathrm{H} \alpha$ and NII $\lambda 4630$. Note that all lines presented exhibit modulations in the magnitude of their emission peaks but only in the stronger Balmer and HeI lines do these modulations coexist with the LT variation, in fact they are superimposed on the declining branch of the LT variation. Note also that the two kinds of variations do not appear to be linked. Even when they spread through one and the same region (in velocity space) they do not interact.

In addition, we would like to highlight the presence of a third peak in the distributions of the variability across P Cygni profiles (right panels of Fig. 4). This peak indicates that the high-velocity region of the red emission wings of the profiles, namely those between +90 and $+230 / 250 \mathrm{~km} \mathrm{~s}^{-1}$, is time variable. Close inspection of several P Cygni profiles enables us to suggest that this variability seems to be due to the occurrence of low-intensity bumps whose position on the wings varies in time.

\subsection{Variability of some "special" lines}

In this section we focus on the lpv of a number of "special" lines in P Cygni's optical spectrum in 1990. These are the resonance lines of $\mathrm{NaI}$, forbidden lines and lines with a pure emission profile. Properties of the lines from the last two groups have recently been studied by Markova \& de Groot (1997).

The resonance lines of $\mathrm{NaI} \lambda \lambda 5890$ (D2), 5896 (D1) are situated on the red emission wing of the HeI $\lambda 5876$ line. During 1990 the profiles of the lines consisted of a blueshifted absorption component in addition to the IS component. Figure 8 (upper panel) displays representative profiles of the D1 and D2 lines. The lower panel of the figure shows the rms deviations of the variations across the profiles for the entire data set. It is evident that both lines have exhibited variations in their troughs. The apparent variability of the IS components certainly has no physical reason and should be ignored since the features are very deep and narrow and, hence, extremely sensitive to small errors in the tracing of the spectra. For example, even when individual spectra in the time series are aligned to within 1 pixel or less, significant deviations occur over this interval within the IS D-lines. To quantify the variability
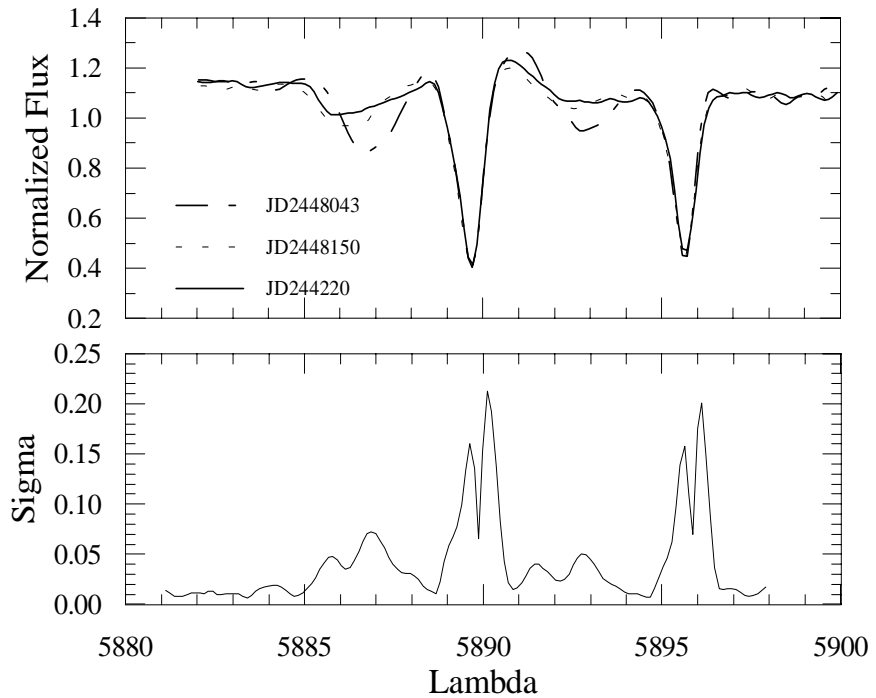

Fig. 8. (upper panel) Representative observational profiles of the D1 and D2 lines of NaI from our data set. (lower panel) The rms deviations for the entire data set as a function of wavelength across the lines

of the stellar components, we measured the FWHM, the normalized flux - i.e. $F_{\min }=\operatorname{Min}\left(F_{\lambda} / F_{\mathrm{c}}\right)$ - and the position - i.e. HRV - of the maximum absorption depth of the absorption trough of the D2-line; the absorption trough of the D1-line is strongly influenced by the red wing of the FeIII $\lambda 5892$ pure emission line. The measurements, which are illustrated in Fig. 9, show that, starting on the first night of the time series until JD 2448109, the properties of the line did not change significantly: the rms deviation amounted to only $2.8 \mathrm{~km} \mathrm{~s}^{-1}$ in HRV, 0.03 in $F_{\text {min }}$ and $0.053 \AA$ in FWHM. After JD 2448109 we observed a systematic increase in the velocity and the width of the trough, which was accompanied by an increase in $F_{\min }$, i.e. by a decrease in the maximum absorption depth. During this episode an absorption bump appeared in the trough at about $-200 \mathrm{~km} \mathrm{~s}^{-1}$. This feature persisted at almost unchanged position for about a month. The variations must be real: in particular, they cannot be artifacts of inconsistent continuum placement or of changes in the strength of the emission wing of the HeI $\lambda 5876$ line, since the rms deviations in the velocity and strength of the nearby IS component amounted to only $2 \mathrm{~km} \mathrm{~s}^{-1}$ and $0.008 \AA$ respectively. No variations similar to either the "swaying" variability or the LT variability were observed.

In order to ascertain whether the established systematic variability of the $\mathrm{NaI}$ resonance lines might be due to the DAC event described in the previous section, we compared the velocity-versus-time behavior of the D2 line with the evolution of the DACs in velocity space. The bottom panel of Fig. 9 emphasizes the good correspondence (in velocity and acceleration) between the two phenomena. Summarizing, we conclude that the behaviour of the NaI resonance lines is more likely dominated (or 


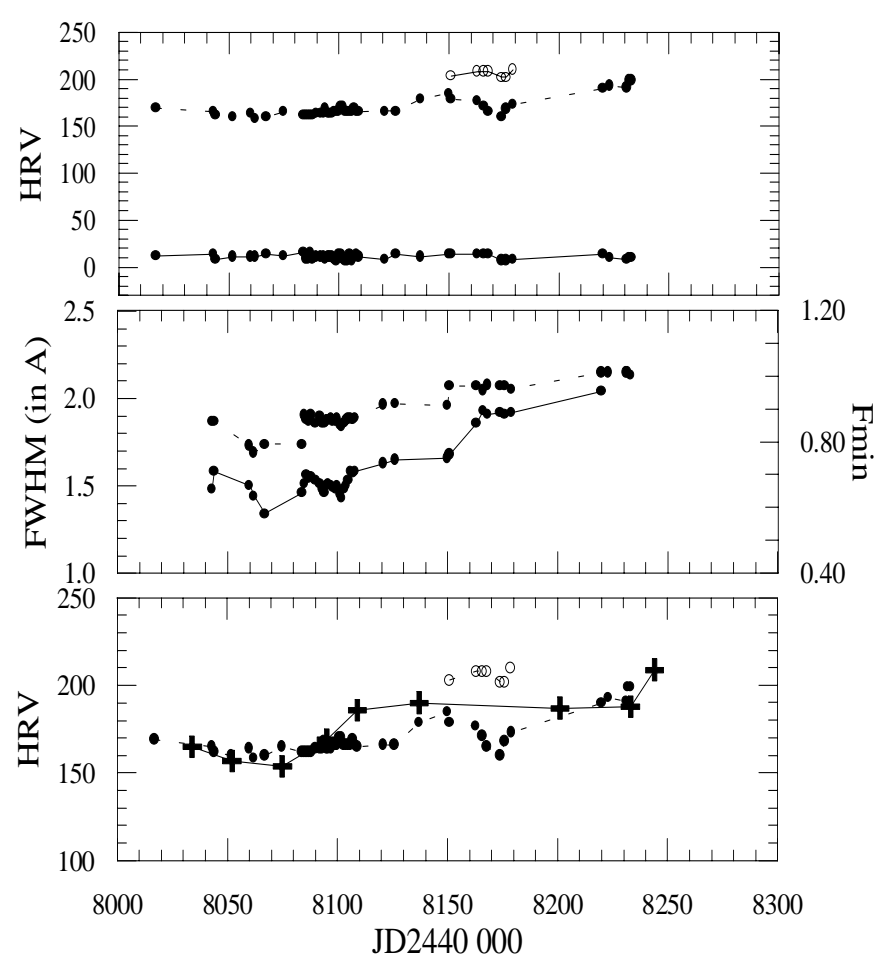

Fig. 9. Variability of measured properties of the absorption trough of the D2 line of NaI. Upper panel: Variations in the velocity of the stellar (dots joined by a dashed line) and IS (dots joined by a solid line) components of the line. Open circles denote the velocity of a bump that occurred in the trough. Middle panel: Variations in the magnitude of the minimum flux, $F_{\min }$ $=\operatorname{Min}\left(F_{\lambda} / F_{\mathrm{c}}\right)$ (dashed line) and in the FWHM (solid line) of the trough. Bottom panel: Comparison between variations in velocity of the D2 line (dots and open circles) and the evolution of the DACs in velocity space (crosses joined by a solid line). All velocities are negative and measured with respect to the Sun (HRV = heliocentric radial velocity)

completely determined) by outward-propagating disturbances of enhanced density, identical to those that cause the appearance of DACs in the intermediate-excitation lines (Sect. 3.1).

Studying the lpv of forbidden lines and lines with pure emission profiles we found that the former did not show any significant variations while the latter did vary, though at a low level of significance. Figure 10 shows the rms deviations across the profiles of the NII $\lambda 5755$ and FeII $\lambda 4285$ forbidden lines (upper panels) as well as of the FeIII $\lambda 5834$ pure emission line (lower panel). The rms deviations across the DIB at $\lambda 5780$ are also shown in order to give the reader an impression about the accuracy of the used technique. Note that the two peaks evident on the upper right plot of the figure do not refer to the forbidden line itself but to the SIII $\lambda 4285$ and OII $\lambda 4289$ lines, which blend with the profile of [FeII] $\lambda 4285$. Because we did not study the properties of the pure emission lines we are not able to specify whether the strengths or the velocities of the profiles vary and what is the pattern of the variations. However, refer-

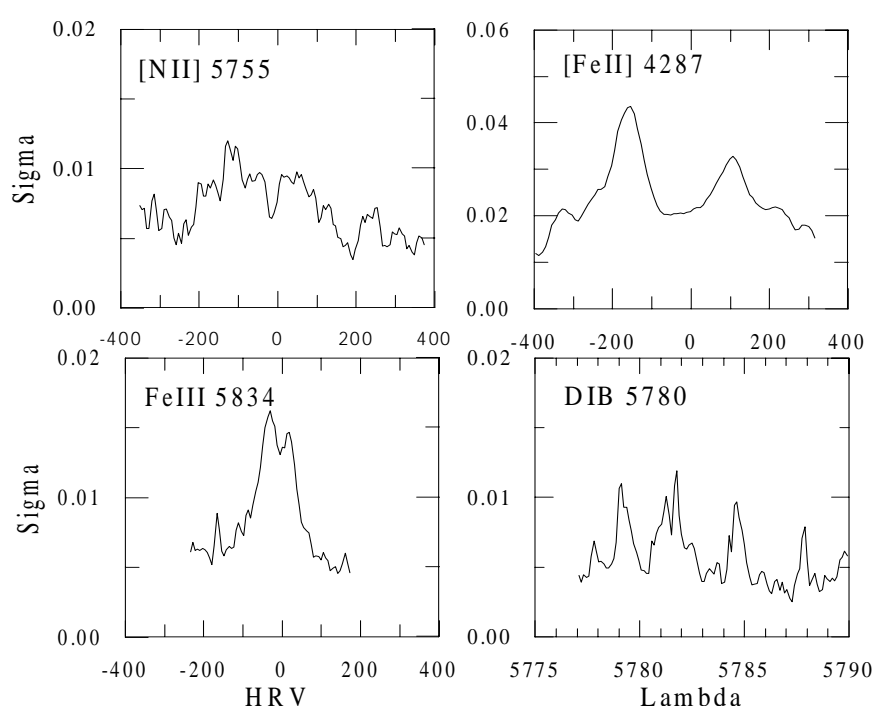

Fig. 10. The rms deviations for the entire data set as a function of velocity (HRV = heliocentric radial velocity) in the [NII] $\lambda 5755$ and [FeII] $\lambda 4285$ forbidden lines (upper panels) and in the pure emission FeIII $\lambda 5834$ line. For comparison the rms deviations across the DIB $\lambda 5780$ are also shown

ring to the result reported by Markova \& de Groot (1997) that the NII and FeIII pure emission lines in P Cygni's optical spectrum seem to form in the inner wind, we suggest that these lines probably show variations similar to the "swaying" variability.

Forbidden lines in P Cygni's optical spectrum presumably originate at large distances from the star (about $100 R_{*}$ ) where the wind has already reached its terminal velocity $\left(V_{\text {inf }}=230 \mathrm{~km} \mathrm{~s}^{-1}\right.$; Stahl et al. 1991; Israelian \& de Groot 1992; Markova \& de Groot 1997) since their profiles are unshifted (in the star's rest frame) and flat-topped. (The only exception is the [NII] $\lambda 5755$ line whose profile is rounded and slightly blue-shifted, thus indicating that the line is formed in an accelerating zone where the velocity of the matter has reached $0.85 V_{\text {inf }}$ (Markova \& de Groot 1997)). The lack of apparent variability for these lines, therefore, implies either that the wind is uniform at this distance or that it is still structured, but the conditions inside the structures do not favour transitions from the relevant metastable levels. Because structures in the wind were observed at even larger distances from the star (Barlow et al. 1994), we suggest that the last hypothesis is the more probable one.

\section{Discussion and conclusions}

Our spectroscopic analysis of P Cygni revealed the presence of four kinds of line-profile variability: LT variability in $\mathrm{HI}$ and HeI lines of relatively high optical depth; red emission-wing variability; DAC-induced variability in the troughs of optical lines of low and intermediate excitation (such as NaI, CaII, HI, HeI and FeIII) and 
"swaying" variability in the troughs and lobes of lines of intermediate and high excitation.

The LT variability consists of a very slow pattern of variation in both the velocity of the absorption cores and the strength of the emission peaks of the strongest HI and HeI lines. Variations in velocity are anti-correlated with changes in the strength of the emission peak. The LT variability is localized only in the outer part of the wind $\left(V \geq 0.82 V_{\text {inf }}\right)$ : neither weaker lines of $\mathrm{HI}$ and $\mathrm{HeI}$ nor lines of higher-excitation ions showed LT variation in velocity or strength. The nature and origin of this kind of variability are still unknown. Recently, Markova et al. (1998) have reported evidence for a LT variation in the brightness and temperature of $\mathrm{P}$ Cygni. The relationship between the LT wind variability and the LT photospheric variability (if any) is the subject of another study (Markova et al. 2000a).

The red-emission-wing variability affects the high-velocity part of the $\mathrm{P}$ Cygni-type profiles, $+90 \leq V \leq+230 / 250 \mathrm{~km} \mathrm{~s}^{-1}$. This variability seems to be caused by low-intensity bumps whose position varies with time. The properties of this phenomenon are still largely unknown. A similar phenomenon may have been observed by Kuss \& Duemmler (1995) who suggested that the bumps are due to light echos from outward-moving dense shells that produce different DAC sequences. Unfortunately, our set of photographic data is not very extended and has large gaps that do not allow any correspondence between the two phenomena to be established.

\subsection{On the nature of DACs}

Our analysis reveals the presence of systematic wind activity in optical lines of low and intermediate excitation (such as NaI, CaII, HI, HeI and FeIII), which takes the form of blueward-migrating DACs. We found evidence of at least one DAC episode in 1990. The same episode was also traced by Israelian et al. (1996) in UV FeIII and FeII lines. The properties of this DAC event are quite similar to the properties of other DAC events at earlier epochs, thus indicating that the mechanism that causes the appearance of DACs in both the UV and optical regions of the spectrum has not changed and was still at work in 1990 as it had been in earlier years. The fact that Stahl et al. (1994) failed to detect any DACs in their 1990 CCD spectra is most likely due to the fact that most lines showing DACs were situated outside the spectral region covered by their observations.

In spite of the great number of observational studies devoted to DACs in P Cygni, our knowledge of the mechanism that causes the phenomenon is still poor. The properties of the DAC events, such as duration, width in velocity space, acceleration, and absorption strength (Markova 1986b; Israelian et al. 1996, present study), indicate that the components are due to large-scale, timedependent structures in the wind. Applying the wellknown InglisTeller relation to the last visible Balmer line with and without line-splitting, we found that the density inside the structures producing the DAC event in 1990 was very high - about one order of magnitude higher than in the ambient wind. At such density the excitation/ionization of the matter is expected to be low. Indeed, our observations showed that the behaviour of the lines with lowest energy in the optical, namely the resonance lines of NaI (Sect. 3.4) and CaII (Markova 1991), was completely governed by the appearance and evolution of DACs. No evidence for variation different from the DACinduced variability was found for these lines.

It seems that the most-frequently invoked model for interpreting the appearance of DACs in P Cygni has been that of outward moving, extended, dense shells (Markova 1986b; Lamers et al. 1985; Van Gent \& Lamers 1986; Israelian et al. 1996). However, since one would expect to observe an increasing emission-line strength due to shell expansion, and since the quotient spectra of our sample did not reveal variations in emission in connection with those in absorption (Sect. 3.1), one is tempted to conclude that the material producing DACs does not seem to exist as a shell. It might well be however that this result has no physical meaning but only reflects the competition between the LT variability and the DAC-induced variability, whose effects on the emission lobes of the lines are contrary to each other (during the period studied). Thus we conclude that DACs in P Cygni may originate from outward moving dense shells.

Alternatively, DACs might be caused by material confined in discrete geometric units, "blobs", "puffs" or "spirals", situated just in front of the stellar disk. Although the hypothesis of moving blops received strong support from radio (Skinner et al. 1997), spectral (Barlow et al. 1994), interferometric (Vakili et al. 1997) and polarimetric (Taylor et al. 1991) observations it meets invincible difficulties in interpreting the properties of the DAC phenomenon. In particular, the apparent regularity of the DAC events, with a quasi-period of about 200 days (Markova 1986b; Israelian et al. 1996), is hardly consistent with the idea of randomly-distributed regions of excess density inferred from polarimetric observations (Taylor et al. 1991). Thus we conclude that DACs in P Cygni are not due to outward moving blobs.

In recent years the model of co-rotating spirals (known as the CIR model: Mullan 1984, 1986; Cranmer \& Owocki 1996) has become popular for the interpretion of the recurrence of DACs in O-type stars (Kaper et al. 1997, 1998). This model seems to be capable of explaining most of the established properties of DACs in P Cygni as well. For example, a spiral-shaped structure would cut across a wide range of projected velocities thus explaining the relatively large width of the DACs in velocity space (about $70 \mathrm{~km}$ $\left.\mathrm{s}^{-1}\right)$. Due to curvature of the spiral, such structure would 
also cause the appearance of a time lag for different velocities, a result that simply appears from a study of the time behaviour of the Balmer progression (Markova 1991). In addition, the established increase and subsequent decrease in the strength of DACs in Balmer lines (Markova 1986a) might also be readily interpreted in terms of a spiral-shaped DAC-forming region which rotates into and out of the line of sight. On the other hand, there are a few results that are not consistent with the CIR model. For instance, if DACs are due to spiral-shaped structures that co-rotate (or nearly co-rotate) with the star, then their character should be strictly periodic (even over a limited time interval) with a cycle length that is determined by both the rotational period of the star, $P_{\text {rot }}$, and the number of co-rotating spirals and, thus, could be comparable to (or be an integer fraction of) $P_{\text {rot }}$. The observations showed that the DAC-induced variability in $\mathrm{P}$ Cygni is not periodic - as one might be tempted to infer from the excellent agreement between the estimates reported in the literature for the recurrence of DACs: $200 \pm 7$ days in 1981-83 (Markova 1986b, optical observations) and $206 \pm$ 11days in 1985-90 Israelian et al. 1996, UV observations) - but occurs on a time-scale that varies from about 150 to about 250 days. Furthermore, from the radius, $76 R_{\odot}$ (Pauldrach \& Puls 1990), and the rotation velocity of the star, $40 \mathrm{~km} \mathrm{~s}^{-1}$ (Israelian 1995), we derive an upper limit to the rotation period of 96 days. Hence, the time-scale of the DACs is longer than the estimated maximum rotation period, $P_{\text {rot }}(\max )$. All this indicates that rotation does not seem to play a fundamental role in determining the recurrence of the DACs in P Cygni.

The recurrence of DACs over an interval of more than 50 years (Markova 1986b) suggests that the structures producing DACs are not a transient feature of the wind but persist over many flow times and are more likely maintained by photospheric processes. This assumption seems also to be supported by the finding that on a few occasions the appearance of a new DAC was accompanied by an increase in the stellar brightness (Israelian et al. 1996). Spectral observations, however, indicate that the structures producing DACs do not seem to be directly linked to the photosphere but originate somewhere up in the wind: no DACs with velocities lower than $90 \mathrm{~km} \mathrm{~s}^{-1}$ have ever been detected (Markova 1986b; Israelian et al. 1996, present study). Thus we conclude that the source of large-scale structures in the wind is more likely coupled with the stellar photosphere but because of some unknown reasons these structures do not develope in the inner part of the wind.

Finally, we note that the longevity of the DAC events, which lasts more than 100 radial flow times of the wind (the characteristic radial flow time of $\mathrm{P}$ Cygni's wind is about 2.8 days), suggests that the structures producing DACs might not represent single mass-conserving features but rather might arise from slowly evolving perturbations through which wind material flows.
In summary, DACs in $\mathrm{P}$ Cygni presumably originate from large-scale, time-dependent, enhanced-density (lowexcitation) perturbations, which develop in the outer part of the wind, $V \geq 0.41 V_{\text {inf }}$ ), but appear to be maintained by photospheric processes. The geometry of the structures is still not clear. It could be either spherically symmetric or curved, like kinks. The recurrence of the DACs does not appear to be related to the stellar rotation.

\subsection{On the nature of the modulations}

The observations showed that the "swaying" variability occurs over a broad range of velocities (negative and positive) within the profile. This variability manifests itself by modulations (like fluctuations above and below a mean level) in the position and intensity of the absorption cores and emission peaks of most lines in the spectrum. Simultaneous variations in emission and absorption-line strength (i.e. EW) were also established.

The properties of the studied lines, together with the trends we found, suggest that the "swaying" variability of the troughs is not just due to the redistribution of a fixed amount of line absorption, as would occur if, e.g., the variability was caused by a macroscopic velocity field alone. Because variations in absorption EW do occur, the process or processes responsible for the observed phenomenon must also alter the number of absorbers, either through changes in the ionization/excitation or in the number density. Although changes in the excitation temperature of the wind, $T_{\text {exc }}$, do occur - estimates derived in terms of a curve-of-growth analysis of 35 NII lines with excitation energies between 18.47 and $23.2 \mathrm{eV}$ showed that the increase in velocity and strength of the lines between JD 2448017 and JD 2448052 (i.e. during the "swaying" variation) was accompanied by a $15 \%$ decrease (about $3000 \mathrm{~K}$ ) in $T_{\text {exc }}$ - it seems unlikely that these variations govern the "swaying" variability of the wind. The fact that optical lines of different excitation and ionization show simultaneous increase/decrease in absorption EW clearly indicates that line-flux (strength) modulations are due to changes in the number density rather than to changes in the ionization/excitation of the wind. Therefore, we suggest that line-flux modulations in P Cygni's optical spectrum more likely originate from time-dependent enhanceddensity structures in the wind.

There are at least two pieces of evidence which indicate that the wind region affected by the modulations should be very extended. We found, first, that the "swaying" variability affects all layers of the supersonic wind starting at its base and up to at least $14 R_{*}$ where the $\mathrm{H} \alpha$ line forms and, second, that the modulations occur in both emission and absorption indicating that the structure producing the modulations should have significant azimuthal extent and could be either spherically symmetric (i.e. shells) or 
spiral-shaped. The hypothesis of spiral-shaped structures in the wind as a possible cause of the modulations implies a picture where spirals rooted in the photosphere co-rotate with the star. Strong evidence in support of this hypothesis would be therefore the establishment of a close relationships between the modulations and the stellar rotation.

It is not clear whether the "swaying" variability of $\mathrm{P}$ Cygni's wind is related to the stellar rotation. The present data turned out to be insufficient and do not enable us to find out whether the established phenomenon is periodic or not. Nevertheless, the pattern of the "swaying" variability appears to be qualitatively similar to the variability found at earlier epochs, thus suggesting that the phenomenon is stable over many years. The time-scale of the modulations, however, is different at different epochs. For example, the modulations in the velocity of the absorption cores of optical lines occurred on a time-scale of 100 to 120 days in 1981 (Markova 1993); of 50 to 60 days in 1982 and 1989 (Kolka 1989, 1991) and of about 100 days in 1990-1991 (present study). These results suggest that the "swaying" variability is more likely caused by a photospheric process that is capable of reproducing itself on a different time-scale. Also, the fact that at some epochs, e.g. 1981, the time-scale of the modulations in velocity was longer than $P_{\text {rot }}(\max )$ suggests that these modulations are probably not caused by co-rotationg structures. The lack of a time lag in the velocity variability of the lines seems to support this assumption, too. On the other hand, it is worthwhile emphasizing that the established line-flux (strength) modulations might be related to the rotation since their time-scale is shorter than $P_{\operatorname{rot}}(\max )$ : in 1989 their time-scale was about 30 days (Kolka 1991) while in 1990 it was about 60 to 70 days.

It is a puzzling aspect of our analysis that the modulations in the velocity of the absorption cores are not obviously related to the modulations in the maximum absorption flux (strength). The two kinds of variations have one and the same pattern of behaviour but their timescales seem to differ. It appears that a similar situation was observed by Kolka (1991) who established variations in emission and absorption-line strength (i.e. EW) on a time-scale about two times shorter than the time-scale associated with simultaneous variations in the velocity of the absorption cores. These results, although rather uncertain due to the uncertainty in the relevant time-scale estimates, imply that the two sorts of modulations might have different origins.

Thus we see that the above results do not enable us to characterize the nature of the modulations completely. It is clearly important to perform a detailed time-series analysis of the modulations on a much more extended data set in order to obtain refined estimates of the relevant timescales and, thus, to make a more definitive assessment of the relationship between different kinds of modu-

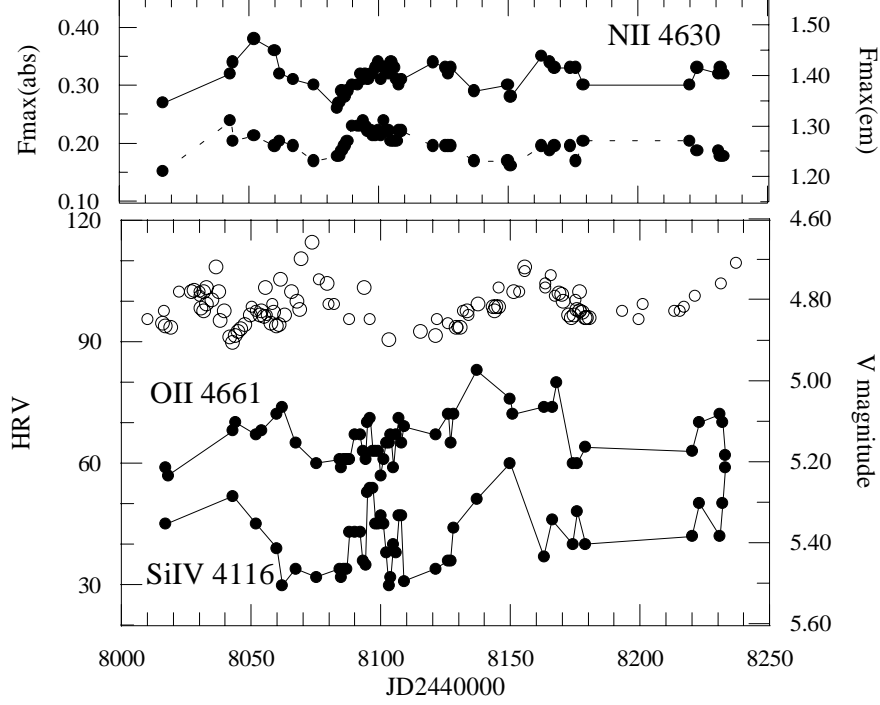

Fig. 11. Variations in the velocity of the absorption cores (lower panel, dots) and in the maximum absorption (upper panel, solid line) and emission (upper panel, dashed line) flux of selected lines formed in the deepest layers of P Cygni's wind, compared with variations in the $V$-band (open circles). All velocities are negative and measured with respect to the Sun $(\mathrm{HRV}=$ heliocentric radial velocity $)$

lations as well as between the modulations and the stellar rotation.

\subsubsection{The modulations and the hypothesis of "photospheric connection"}

In the previous subsection we have suggested, based on indirect evidence, that the modulations of P Cygni's wind might be caused by photospheric processes. The existence of (quasi-)simultaneous photospheric (Percy et al. 1996; Markova et al. 2000b) and spectral observations (Stahl et al. 1991, present study) allows us to test this hypothesis. Attempts to detect a direct coupling between stellarwind variability and photospheric variability have become known as the search for a "photospheric connection".

In Fig. 11 the "swaying" variability in both the velocity of the absorption cores and in the maximum absorption and emission-line flux, $F_{\max }(\mathrm{abs})$ and $F_{\max }(\mathrm{em})$, of selected high-excitation lines is compared with changes in the stellar brightness. The photometric data presented are taken from Markova et al. (2000b). It is apparent from this figure that:

(i) the modulations in the $F_{\max }(\mathrm{abs})$ and $F_{\max }(\mathrm{em})$ tend to be anti-correlated with the variations in the brightness; (ii) the modulations in the velocity of the absorption cores appear to be correlated with changes in the brightness with a probable phase lag.

Notice that most of the time the fluctuations in $F_{\max }(\mathrm{em})$ have been accompanied by similar fluctuations in $F_{\max }(\mathrm{abs})$, a fact that completely rules out the suspicion 
one may have, when looking at the apparent anticorrelation between variations in the NII $\lambda 4630$ luminosity and in the $V$-band, that the line-flux variability is only a reflection of the changing continuum. It is also worth noting that Markova et al. (2000b) have established variations in the stellar temperature that correlate with changes in the $V$-band, so that when the brightness increases the temperature decreases.

The above allows us to suggest that the "swaying" variability of $\mathrm{P}$ Cygni's wind is probably triggered by photospheric processes. This assumption seems to be supported also by two additional results. First, the finding that the time-scale of the "swaying" variability equals at least $21 t_{\text {flow }}$ (during the studied period) together with the fact that the modulations last for more than $82 t_{\text {flow }}$, indicate that these modulations are not transient features of the wind, but persist for many flow times and seem to be caused by photospheric processes. And, second, the radialvelocity measurements convincingly showed that the modulations affected the deepest layers of the supersonic wind down to its base. Evidence for a possible relationship between variations in the wind and in the stellar parameters was reported by different investigators. For example, de Groot (1990) noted that an increase in the star's brightness seemed to be accompanied by a decline in the strength of $\mathrm{H} \alpha$ emission and by an increase in $B-V$. A probable correlation between variations in the velocity of the absorption cores and changes in the brightness was suggested by Kolka (1991). All this suggests that the "swaying" variability of P Cygni's wind is more likely coupled to the photosphere.

If the modulations of P Cygni's wind are indeed triggered by photospheric processes what could these be? On the one hand, the position of P Cygni on the HR-diagram falls within the predicted instability strip for strange-mode oscillations (Kiriakidis et al. 1993) thus suggesting that non-radial pulsations (NRP) of strange-mode oscillations could be a possible cause for the ST photometric variability of the star. On the other hand, Lamers et al. (1998) have recently argued that the microvariability of LBVs appears to be very similar to the variability of normal B-supergiants and slowly-pulsating B-stars - both due to NRPs of gravitational-mode oscillations. The authors showed further that the microvariability of LBVs can also be explained by means of NRPs of g-modes of low $l$. Thus we suggest that a possible cause for the ST photometric variability (i.e. for microvariability) of $\mathrm{P}$ Cygni are NRPs of either s-mode or g-mode oscillations. Small variations in stellar parameters could lead to variations in the properties of the wind due to the postulated sensitivity of P Cygni's wind (Pauldrach \& Puls 1990).

Summarizing, we conclude that the "swaying" variability of P Cygni's optical wind is most likely triggered by either s-mode or g-mode NRPs in the photosphere.

\subsection{Are the modulations and DACs linked to each other?}

There are at least two pieces of evidence suggesting that the modulations and the DACs are probably not linked to each other. In particular, it seems unlikely that the modulations of the troughs are caused by the DACs themselves. First, the time-scales of the two phenomena are too different to suggest they might be physically coupled and, second, there are lines (e.g. the resonance lines of $\mathrm{NaI}$ and CaII) that show DACs but do not show modulations in velocity or strength of their troughs.

On the other hand, Markova (1993) found that on two different occasions in 1981 the increase in the velocity of the absorption cores of high-excitation optical lines (i.e. the rising branch of a modulation in velocity) seemed to precede the appearance of DACs in low-excitation lines. Unfortunately, due to the very limited number of available photographic spectra, we were not able to look for such a relationship in 1990. According to Israelian et al. (1996), however, a new DAC event should appear around JD 2448147. From a casual inspection of the data presented in Fig. 6 it seems that this event corresponds to a maximum in the velocity curve of the "swaying" variability, thus supporting the result reported by Markova (1993). These results imply that a possible relationship between the modulations in the velocity of the absorption cores of the lines and the DACs cannot be excluded. If this is true then some additional factor would be needed to explain why structures of sufficient strength to produce observable DACs do not occur during every cycle of the modulations.

The above indicates that the available observations are insufficient to enable us to resolve completely the problem of a possible relationship between the modulations and the DACs. The establishment of such a relationship is a very difficult task since - due to the long time-scale of the DAC phenomena - it demands a very long-term monitoring campaign with high temporal and spectral resolution. Another requirement is that the observations must cover a spectral window that is going down to about $3500 \AA$ since in the higher members of the Balmer and HeI series the modulations and the DACs should coexist.

\subsection{A few concluding remarks}

In the previous subsection we have noted that DACs in $\mathrm{P}$ Cygni are not obviously connected to the stellar rotation. This is what distinguishes the wind variability of this Btype hypergiant from the variability of O-star winds, for which the observations clearly indicate a close relationship between the recurrence of DACs and the rotational period (Kaper et al. 1996, 1997, 1998). In addition, O-star winds do not give evidence for continuous modulations. However, P Cygni's wind variability is similar to that of another B supergiant, HD 64760. DACs in this star appear 
on a timescale that is two to three times longer than the estimated rotational period (Prinja et al. 1995). A further comparison of our results with those published by Prinja et al. (1995) and Fullerton et al. (1997) for HD 64760 showed that the wind variability of these B-type luminous stars appears to be qualitatively similar in several aspects. For example, both stars exhibit line-flux modulations that extend over the troughs and the lobes of the lines and occur on time-scales that vary slowly with time: HD 64760 in UV lines, P Cygni in optical lines; both stars show DACs which recur on time-scales that are not related to the rotation: HD 64760 in UV lines, P Cygni in UV and optical lines; in both stars DACs consist of a "slower" pattern of wind variability on which a "faster" pattern caused by line-flux modulations is superimposed; in both stars the modulations and the episodic DACs do not interact in an obvious manner; both stars give suggestive evidence for a "photospheric connection" either through variations in low-velocity $(v \simeq 0)$ wind layers (HD 64760, Massa et al. 1995; P Cygni, present study) or through close relationships between spectral and photometric variations (P Cygni, Markova et al. 2000a).

The periodic modulations in HD 64760 have been interpreted in terms of few corotating spirals that modulate the optical depth of the wind (Fullerton et al. 1997). Since the basic physical ingredients responsible for the appearance of the spirals - photospheric variability and stellar rotation - occur very commonly among OB stars the modulations are expected to be a common signature of wind variability of many early type stars. To explain the lack of observational evidence for rotational modulation of Ostar winds Fullerton et al. (1997) supposed that due to the smaller curvature of the spirals the structures are intrinsically more difficult to detect in these stars. (Note that the winding of a kinematic spiral is largely determined by the ratio $V_{\text {rot }} / V_{\text {inf }}$, which is expected to be smaller for Ostars - due to their systematically higher wind terminal velocities - than for B-stars.)

In this connection it is worth emphasizing that P Cygni and HD 64760 strongly differ in their stellar and wind parameters, especially rotational velocity and wind terminal velocity: while the former is a slowly rotating star $\left(v \sin i=40 \mathrm{~km} \mathrm{~s}^{-1}\right)$ with a slow wind $\left(V_{\mathrm{inf}}=230 \mathrm{~km}\right.$ $\mathrm{s}^{-1}$, Markova \& de Groot 1997; Stahl et al. 1991), the latter is an extremely rapid rotator $\left(v \sin i=238 \mathrm{~km} \mathrm{~s}^{-1}\right.$ Hoffleit \& Jaschek 1982) emitting a fast stellar wind ( $V_{\text {inf }}$ $=1500 \mathrm{~km} \mathrm{~s}^{-1}$, Massa et al. 1995), The two stars however have almost the same values of $V_{\text {rot }} / V_{\text {inf }}: 0.16$ for HD 64760 and 0.17 for P Cyg. This result together with the noted qualitative similarity in their wind variability seem to support the assumption of Fullerton et al. about the essential role that the ratio $V_{\text {rot }} / V_{\text {inf }}$ may play in determining the properties of wind variability of early type stars.

The fact that wind variability consisting of continuous modulations and DACs was detected in $\mathrm{P}$ Cygni through optical observations emphasizes the importance of longterm ground-based surveys as a powerful tool for the study of wind variability of stars with at least stronger stellar winds. This finding is quite comforting and promising especially under the tangible absence of the IUE satellite services nowadays.

Acknowledgements. I wish to thank Drs. Mart de Groot and Garik Israelian for their detailed comments on this work. I am also grateful to Dr. Otmar Stahl and his collegues at the Landessternwarte, Heidelberg for making their observations of P Cygni available by publishing them in The Journal of Astronomical Data. This work was supported by NSF to MES trough grants No. F-813/1998.

\section{References}

Barlow M.J., Drew J.E., Meaburn J., Massey R.M., 1994, MNRAS 268, L29

Cranmer S.R., Owocki S.P., 1996, ApJ 462, 469

de Jager C., 1980, The Brightest Stars, 60

de Groot M., 1969, Bull. Astron. Inst. Netherl. 20, 225

Fullerton A.W., Gies D.R., Bolton C.T., 1996, ApJS 103, 425

Fullerton A.W., Massa D.L., Prinja R.K., et al., 1997, A\&A 327,699

Henrichs H.F., Kaper L., Nichols J.S., 1994, A\&A 285, 565

Hayes D.P., 1985, ApJ 289, 726

Hoffleit D., Jaschek C., 1982, The Bright Star Catalogue, 4th edition, Yele University Observatory, New Haven

Israelian G., de Groot M., 1992, in Nonisotropic and Variable Outflows from Stars, Drissen L., Leitherer C., Nota A. (eds.) ASPC Ser. 22, 356

Israelian G., 1995, A\&A 300, 834

Israelian G., de Groot M., Parker J.Wm., Sterken C., 1996, MNRAS 937, 230

Israelian G., de Groot M., 1999, SSR 90, 493

Kaper H.E., et al., 1996, A\&AS 116, 257

Kaper H.E., Henrichs H.F., Fullerton A.W., et al., 1997, A\&A 327,281

Kaper H.E., Henrichs H.F., Nichols J.S., Telting J.H., 1998, ESO preprint No. 1311

Kiriakidis M., Pricke K.J., Glatzer W., 1993, MNRAS 264, 50

Kolka I., 1989, in "Physics of Luminous Blue Variables", IAU Coll. 113, Davidson K., Moffat A.F.J., Lamers H.J.G.L.M. (eds.). Kluwer, Dordrecht, p. 265

Kolka I., 1991, Workshop on "The atmospheres of early-type stars", Heber U., Jeffery C.S. (eds.), Lect. Notes Phys. 401, 125. Springer, Berlin

Kuss C., Duemmler R., 1995, Astron. Gess., Abst. Ser., No. 9, 136

Lamers H.J.G.L.M., 1986, in "Luminous Stars and Associations in Galaxies", IAU Symp. 116, de Loore C. and Willis A. (eds.). Reidel, Dordrecht

Lamers H.J.G.L.M., de Groot M., Cassatella A., 1983, A\&A 128,299

Lamers H.J.G.L.M., Bastiaanse C., Aerts C., Spoon H.W.W., 1998, A\&A 335, 605

Luud L., 1967a, Sov. Astr. 11, 211

Luud L., 1967b, Dokl. Akad. Nauk Armenianskoi SSR 3, 379

Markova N., 1986a, A\&SS 123, 5 
Markova N., 1986b, A\&A 162, L3

Markova N., 1991, Ph.D. Thesis, Institute of Astronomy, National Astronomical Observatory

Markova N., 1993, A\&A 273, 555

Markova N., de Groot M., 1997, A\&A 326, 1111

Markova N., Scuderi S., de Groot M., 1998, IAU Coll. 169, Heidelberg, Germany

Markova N., Morrison N., de Groot M., Kolka I., 2000a (in preparation)

Markova N., Scuderi S., de Groot M., Panagia N., 2000b (submitted to A\&A)

Massa D., Prinja R.K., Fullerton A.W., 1995, ApJ 452, 842

Mullan D.J., 1984, ApJ 283, 303

Mullan D.J., 1986, A\&A 165, 157

Percy J.R., Attard A., Szczesny M., 1996, A\&ASS 177, 255

Pauldrach A.W.A., Puls J., 1990, A\&A 237, 409
Prinja R.K., Balona L.A., Bolton C.T., et al., 1992, ApJ 390, 266

Prinja R.K., Massa D., Fullerton A.W., 1995, ApJ 452, L62

Prinja R.K., Fullerton A.W., Crowther P.A., 1996, A\&A 311, 264

Skinner C.J., Exter K.M., Barlow M.J., et al., 1997, MNRAS 288, L7

Stahl O., Mandel H., Szeifert Th., et al., 1991, A\&A 244, 467

Stahl O., Wolf B., Gäng Th., et al., 1994, A\&AS 107, 1

Taylor M., Nordsieck R.E., Schulte-Ladbeck R., et al., 1991, AJ 102, 1197

Vakili F., Mourard D., Bonneau F., et al., 1997, A\&A 323, 183

Van den Oord G.H.J., Waters L.B.F.M., Lamers H.J.G.L.M., et al., 1985, in "Radio Stars", Hjellming R.M., Gibson D.M. (eds.). Reidel, Dordrecht, p. 111

Van Gent, Lamers H.J.G.L.M., 1986, A\&A 158, 335 